\title{
Recent Results and Future Plans of the MoEDAL Experiment
}

\author{
Arka Santra* on behalf of the MoEDAL Collaboration \\ Instituto de Fisica Corpuscular, CSIC and Universidad de Valencia, Parque Cientifico, \\ C/Catedratico Jose Beltran, 2, 46980, Paterna, Espana. \\ E-mail: santra.arka@ific.uv.es
}

\begin{abstract}
MoEDAL is an LHC experiment designed to search for anomalously ionizing messengers of new physics such as magnetic monopoles or massive (pseudo-)stable charged particles. These particles are predicted to exist in many models beyond the Standard Model. MoEDAL's physics program yields insights into such foundational questions as: are there extra dimensions or new symmetries; what is the mechanism for the generation of mass; does magnetic charge exist; and what is the nature of dark matter. We will present the results from the MoEDAL detector on magnetic monopole and highly ionizing electrically charged particle production. In conclusion, progress on the installation of MoEDAL's MAPP (MoEDAL Apparatus for Penetrating Particles) sub-detector prototype will be briefly discussed.
\end{abstract}

European Physical Society Conference on High Energy Physics - EPS-HEP2019 -

10-17 July, 2019

Ghent, Belgium

${ }^{*}$ Speaker. 


\section{Introduction}

In Nature, even though the isolated electric charge exists, the magnetic monopole, the magnetic equivalent of electric charge, has not been conclusively detected by any experiment to date. Due to the absence of magnetic monopoles, the Maxwell's equations are asymmetric in electrical and magnetic charge. The presence of magnetic monopole can alter this scenario making Maxwell's equation symmetric under the rotation in $(\vec{E}, \vec{B})$ plane where $\vec{E}$ is the elctric field and $\vec{B}$ is the magnetic field.

There are many experiments which look for signatures of the magnetic monopole. One such experiment is the Monopole and Exotics Detector at the LHC (MoEDAL) [1]. This detector is mostly passive and consists of passive tracking detectors (which uses Nuclear Track Detectors or NTDs in short), magnetic monopole trappers (MMT) made of Aluminium (Al) bars and small TimePix pixel device array for monitoring real-time radiation. The specific goal of MoEDAL is to detect highly ionizing avatars of new physics. This detector, due to its design, is insensitive to the Standard Model (SM) backgrounds. A full GEANT4 [2] model of MoEDAL is shown in Figure 1.

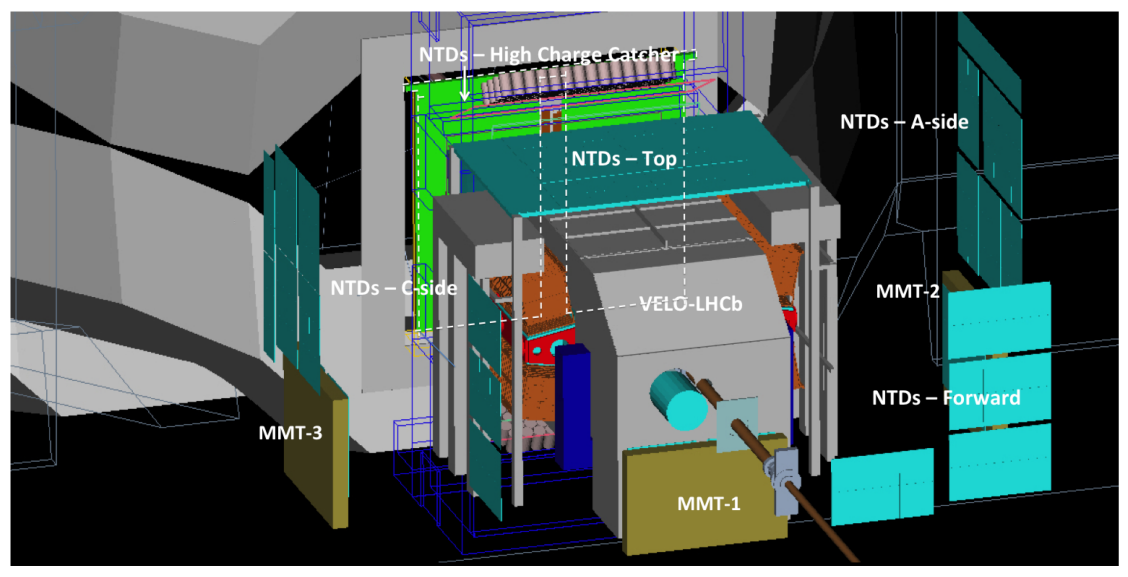

Figure 1: A visualization of the MoEDAL Detector using GEANT4's PANORAMIX package [3].

\section{The MoEDAL Detector}

The MoEDAL detector employs unconventional methodologies in order to look for new physics. This largely passive detector is situated in the Vertex Locator (VELO) cavern of LHCb experiment at Interaction Point (IP) 8. The NTD behave like a giant camera which records the tracks of highly ionizing particles. The MMT traps the particle messengers of new physics, such as magnetic monopoles, for further study.

\subsection{The Nuclear Track Detectors}

The NTD subsystem is made of array of plastic NTD stacks, each of dimension $25 \times 25 \mathrm{~cm}^{2}$. Each of the stack contains 3 CR39 and 3 MAKROFOL plasic NTD sheets. When a highly ionizing particle (HIP) passes through the NTD stacks, the track damages the long chain of the molecular bonds of the plastic. After the NTD detectors are exposed for a year, they are removed for etching 
under controlled conditions. The etching removes the plastic along the damage trail caused by HIP tracks revealing the etch pits along the tracks. The size and depth of the etch pits depend on the charge of the particles passing through the NTDs. The etched NTDs are then scanned through optical scanning microscope system developed by the University of Helsinki to search for the position and size of the etched pits in the NTDs.

\subsection{The Magnetic Monopole Trapper}

The Magnetic monopole trapper, as the name suggests, is used to trap the magnetic monopoles and similar highly ionizing beyond SM particles. This subdetector consists of $794 \mathrm{~kg}$ of Al trapping volumes deployed around the VELO cavern of IP8. A photo of the deployment of the MMT stack is shown in Figure 2. After the exposure, the MMT Al bars are cut into pieces and are passed through the SQUID (Superconducting Quantum Interference Device) magnetometer kept at ETH Zurich. If there is a persistent current in the magnetometer for one Al sample, this would show the presence of the monopole in that $\mathrm{Al}$ sample. To reduce the statistical uncertainty related to the behaviour of the SQUID magnetometer, an Al sample is passed through the magnetometer more than once. The calibration of SQUID magnetometer is done with the help of very long thin solenoids mimicking the response of a monopole. This magnetometer has a resolution of $0.1 g_{\mathrm{D}}$, with $g_{\mathrm{D}}$ being the Dirac charge.
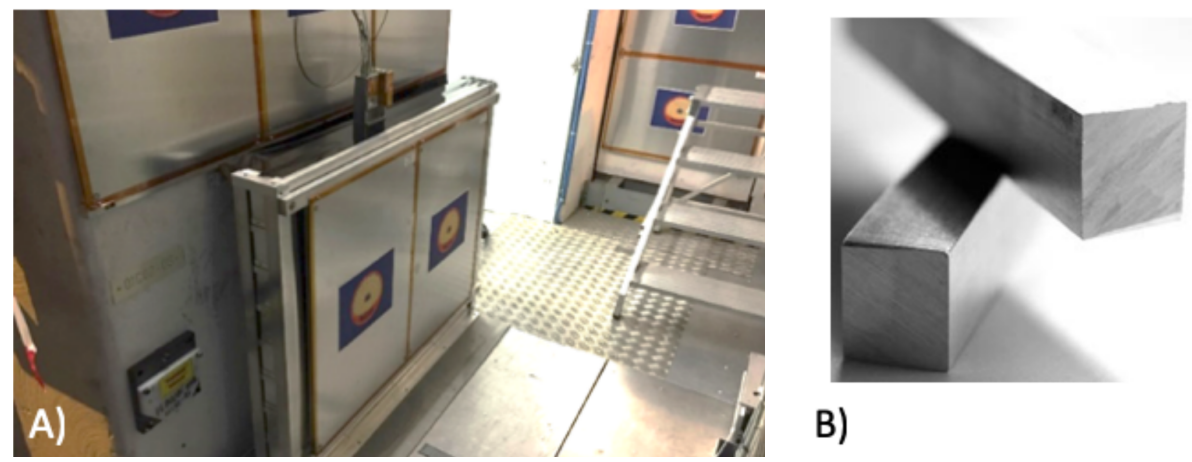

Figure 2: (A) The C-side (negative $\mathrm{x}$-coordinate) MMT detector stack. Other two MMT arrangements are similar. (B) The aluminium bars comprising the MMT subdetector.

\section{Results}

The MoEDAL experiment first published its physics analysis paper in 2016 based on the data taken at the collision energy of $8 \mathrm{TeV}$ [4]. In the most recent searches for monopoles with this detector [5], the data were taken at a center of mass energy of $13 \mathrm{TeV}$ (Run 2) with integrated luminosity of $4.0 \mathrm{fb}^{-1}$. In this search, photon fusion production mechanism of monopole production was taken into account to interpret the result, which is done for the first time at the LHC. In this paper, the search result was interpreted with different production scenarios of monopole, such as velocity dependent and independent photon monopole coupling, different spins of monopole (spin 0,1/2 and 1) and Drell-Yan and photon fusion production mechanism. A detailed theoretical study comparing Drell-Yan and photon fusion mechanism and velocity dependent and independent photon monopole coupling can be found elsewhere [6]. 
Table 1: $95 \%$ C.L. mass limits for monopole pair direct production in models of spin-0, spin- $1 / 2$ and spin-1 in LHC $p p$ collisions. The present results are interpreted for Drell-Yan and combined DY and photon-fusion production with both $\beta$-independent and $\beta$-dependent photon monopole couplings.

\begin{tabular}{|c|c|c|c|c|c|c|}
\hline \multirow{2}{*}{$\begin{array}{l}\text { Process / } \\
\text { coupling }\end{array}$} & \multirow{2}{*}{ Spin } & \multicolumn{5}{|c|}{ Magnetic charge $\left[g_{\mathrm{D}}\right]$} \\
\hline & & 1 & 2 & 3 & 4 & 5 \\
\hline & & \multicolumn{5}{|c|}{ 95\% C.L. mass limits $[\mathrm{GeV}]$} \\
\hline DY & 0 & 790 & 1150 & 1210 & 1130 & - \\
\hline DY & $1 / 2$ & 1320 & 1730 & 1770 & 1640 & - \\
\hline DY & 1 & 1400 & 1840 & 1950 & 1910 & 1800 \\
\hline DY $\beta$-dep. & 0 & 670 & 1010 & 1080 & 1040 & 900 \\
\hline DY $\beta$-dep. & $1 / 2$ & 1050 & 1450 & 1530 & 1450 & - \\
\hline DY $\beta$-dep. & 1 & 1220 & 1680 & 1790 & 1780 & 1710 \\
\hline $\mathrm{DY}+\gamma \gamma$ & 0 & 2190 & 2930 & 3120 & 3090 & - \\
\hline $\mathrm{DY}+\gamma \gamma$ & $1 / 2$ & 2420 & 3180 & 3360 & 3340 & - \\
\hline $\mathrm{DY}+\gamma \gamma$ & 1 & 2920 & 3620 & 3750 & 3740 & - \\
\hline $\mathrm{DY}+\gamma \gamma \beta$-dep. & 0 & 1500 & 2300 & 2590 & 2640 & - \\
\hline $\mathrm{DY}+\gamma \gamma \beta$-dep. & $1 / 2$ & 1760 & 2610 & 2870 & 2940 & 2900 \\
\hline $\mathrm{DY}+\gamma \gamma \beta$-dep. & 1 & 2120 & 3010 & 3270 & 3300 & 3270 \\
\hline
\end{tabular}

In the full Run 2 search [5], no candidate remained after the scanning procedure was completed and magnetic charges equal to or above $1 g_{\mathrm{D}}$ were excluded for all $\mathrm{Al}$ samples. The cross-section upper limits as low as $11 \mathrm{fb}$ were set which is an improvement over the previous limit of $40 \mathrm{fb}$. Mass limits in the range of $1500 \mathrm{GeV}-3750 \mathrm{GeV}$ were placed for magnetic charges up to $5 g_{\mathrm{D}}$ and monopole spins of $0,1 / 2$ and 1 . This is the strongest limits to date at a collider experiment for charges $2-5 g_{\mathrm{D}}$. The summary of the mass limits is presented in Table 1.

\section{Beyond Magnetic Monopole Searches}

Apart from the monopole searches, MoEDAL experiment can search for highly electrically charged objects as well as a few specific supersymmetric scenarios having (meta-)stable slowly moving singly charged particles in the final state [7]. In such scenarios, NTD system of the MoEDAL detector is used to detect charged particles which are above the detection threshold of the most sensitive (CR39) component $(z / \beta \geq 5)$. Preliminary study with simulation shows the sensitivity of MoEDAL versus ATLAS and CMS detectors, for various long-lived particles in the supersymmetric models. An example of a competitive result from MoEDAL and CMS is shown in Figure 3 where gluino $(\tilde{g})$ pair production with $\tilde{g}$ decaying to long-lived neutralino $\left(\tilde{\chi}_{1}^{0}\right)$ decaying to metastable stau $\left(\tilde{\tau}_{1}\right)$ and a $\tau$ lepton is considered. The mass spitting for this model is $30 \mathrm{GeV}$ for $\tilde{\chi}_{1}^{0}$ and $\tilde{g}$ and $300 \mathrm{GeV}$ for $\tilde{\chi}_{1}^{0}$ and $\tilde{\tau}_{1}$. For longer lived particles having $z / \beta \geq 5$ in more complex topologies, MoEDAL can provide added value to the search.

\section{Future Developments}

MoEDAL has a number of plans for the future, both in detector upgradation and adding new 


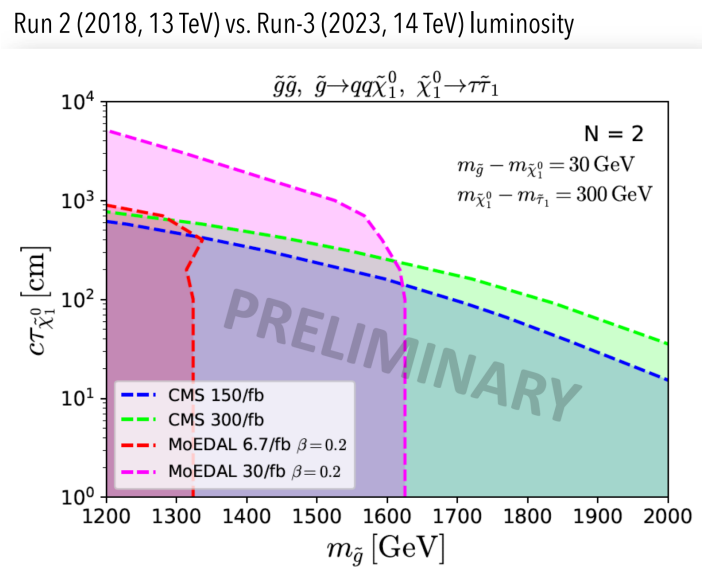

Figure 3: The discovery reach of MoEDAL requiring two signal events versus the 95\% CL exclusion plot in the $\tilde{g}$ mass vs $\tilde{\chi}_{1}^{0}$ lifetime plane. Here the luminosities from both Run $2(13 \mathrm{TeV})$ and Run $3(14 \mathrm{TeV})$ were compared [7].

physics searches.

\subsection{CMS Beam Pipe}

The beam pipes of a collider experiment are the most directly exposed material and it can trap many highly ionizing particles like high magnetically charged monopoles which may not reach the subsequent detector systems down the line. To search for those particles, MoEDAL is using the Run-1 beam pipe of the CMS experiment. The CMS beam pipe (made of beryllium) was cut into pieces and then those pieces were scanned through the SQUID magnetometer in the spring and summer of 2019. At present, a simulation study is ongoing to infer about the acceptance of the beam pipe.

\subsection{MAPP}

For the Run-3 program of the LHC, MoEDAL is preparing for a new subdetector named MoEDAL Apparatus for Penetrating Particles (MAPP) [3] which will be installed in the UGC1 gallery near the existing MoEDAL/LHCb detectors. The motivation behind this detector is to search for fractionally charged particles having charge as low as $10^{-3}$ of electric charge (with the milli-charged particle detector or MAPP-mQP made of scintillator bars) and long-lived weakly interacting neutral particles assumed to be messengers of new physics (with the long-lived particle detector system or MAPP-LLP made of three pairs of hodoscopes). A schematic diagram of MAPP is shown in Figure 4. A prototype of the mQP detector ( $\sim 10 \%$ of the original system) is already in place since 2017.

\section{Conclusion}

The MoEDAL experiment has placed the strongest limits on multiply charged magnetic monopole production. This experiment seeks to continue its data-taking during LHC's Run 3. The experiment is now pushing its boundaries and wants to make its presence strongly in the searches of different 
long-lived supersymmetric scenarios as well as exotic particles like mQP and long-lived neutral particles.

\section{Acknowledgement}

The author acknowledges support by the Generalitat Valenciana through a MoEDAL-supporting agreement with the University of Valencia and the excellence grant PROMETEO-II/2017/033, by the grant PGC2018-094856-B-I00 (MCIU/AEI/FEDER, UE), and by the Severo Ochoa Excellence Centre Project SEV-2014-0398.

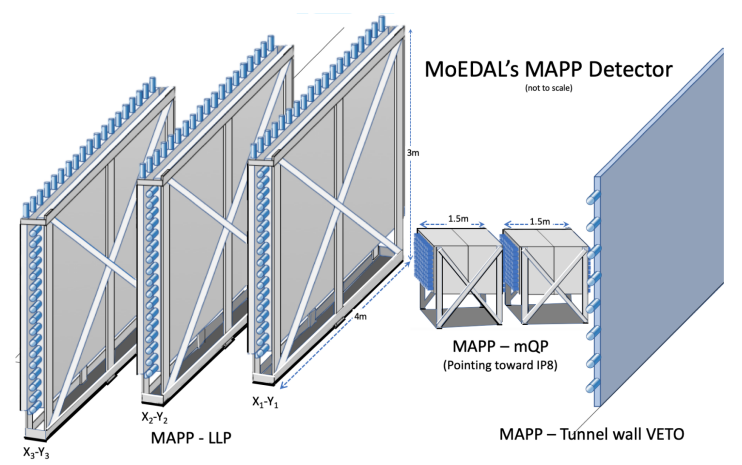

Figure 4: A schematic diagram of MAPP detector showing milli-charged particle detector (mQP) and longlived particle detector (LLP) subdetectors.

\section{References}

[1] Pinfold, J.L.; The MoEDAL Collaboration. Technical Design Report of the MoEDAL Experiment, CERN-LHCC-2009-006, MoEDAL-TDR-001. Available online: http://inspirehep.net/record/1299494/ (accessed on 04 October 2019)

[2] S. Agostinelli et al. [GEANT4 Collaboration], Nucl. Instrum. Meth. A 506, 250 (2003). doi:10.1016/S0168-9002(03)01368-8

[3] Pinfold, J.L.,Universe 2019, 5(2), 47; https://doi.org/10.3390/universe5020047

[4] The MoEDAL collaboration, Acharya, B., Alexandre, J. et al., J. High Energ. Phys. (2016) 2016: 67, https://doi.org/10.1007/JHEP08(2016)067

[5] The MoEDAL Collaboration, B. Acharya et al., Phys. Rev. Lett. (2019) 123, 021802, https://doi.org/10.1103/PhysRevLett.123.021802

[6] Baines, S., Mavromatos, N.E., Mitsou, V.A. et al. Eur. Phys. J. C (2018) 78: 966. https://doi.org/10.1140/epjc/s10052-018-6440-6.

[7] K Sakurai, D Felea, J Mamuzic, N E Mavromatos, V A Mitsou, ,J L Pinfold, R Ruiz de Austri, A Santra and O Vives, IFIC/19-16KCL-PH-TH/2019-34, arXiv:1903.11022v1 [hep-ph] 26 Mar 2019. 